\title{
Studying Static, Dynamic and Transport Properties of $\mathrm{Mg}_{3} \mathrm{Bi}_{2}$
}

\author{
F. KAYADIBI ${ }^{a, *}$, S.D. GÜNAY ${ }^{b}$ AND Ç. TAŞSEVEN ${ }^{b}$
}

${ }^{a}$ Department of Physics, Science and Art Faculty, Kocaeli University, Umuttepe Campus, 41380, Kocaeli, Turkey

${ }^{b}$ Department of Physics, Faculty of Science, Yildiz Technical University, Davutpaşa Campus, 34210, Istanbul, Turkey

(Received January 19, 2015)

\begin{abstract}
In this study, new potential parameters for $\mathrm{Mg}_{3} \mathrm{Bi}_{2}$ are proposed which is the Born-Mayer-Huggins type potential. Static, dynamic and transport properties are studied for this material from $300 \mathrm{~K}$ up to $1600 \mathrm{~K}$ with classical molecular dynamics simulation. Mechanical properties; like elastic constants $\left(C_{11}, C_{12}, C_{13}, C_{33}, C_{44}\right)$, bulk modulus and shear modulus are found. All these data are compared with the limited number of experimental and first-principle studies. Our results give a good description of the $\mathrm{Mg}_{3} \mathrm{Bi}_{2}$ system: lattice constants, $\alpha \rightarrow \beta$ transition temperature, melting temperature, diffusion coefficient, density and mechanical properties are promising.

DOI: 10.12693 /APhysPolA.128.440

PACS: 83.10.Rs, 34.20.Gj, 66.30.H-, 66.30.Fq, 62.20.-x, 65.40.-b
\end{abstract}

\section{Introduction}

Scientists have investigated the alloys with magnesium in recent years for its technological applications in automobile and aerospace industry because of its low density and high strength $[1,2] . \mathrm{Mg}_{3} \mathrm{Bi}_{2}$ has been used in technology at various fields, such as Li-ion batteries [3], thermoelectric element [4] and photoconduction [5]. Some groups have been focused on this material; the optical and semiconducting nature of $\mathrm{Mg}_{3} \mathrm{Bi}_{2}$ had been investigated by Watson et al. [6]. Bi ion concentration in magnesium alloys had been studied and their effect on heat resistance had been explained by Yuan et al. [7, 8]. Solid $\mathrm{Mg}_{3} \mathrm{Bi}_{2}$ is a metal and the liquid $\mathrm{Mg}_{3} \mathrm{Bi}_{2}$ is a semiconductor so that material has a metal-nonmetal transition [9]. These studies have made it more interesting to work on $\mathrm{Mg}_{3} \mathrm{Bi}_{2}$ molecule.

The structure of $\alpha-\mathrm{Mg}_{3} \mathrm{Bi}_{2}$ is a hexagonal anti- $\mathrm{La}_{2} \mathrm{O}_{3}$ type structure with the space group of $P 3 m 1$ [10]. The $\alpha$ phase of $\mathrm{Mg}_{3} \mathrm{Bi}_{2}$ exists at room temperature up to $976 \mathrm{~K}$ and $\beta$ phase exists in the range $976-1094 \mathrm{~K} . \mathrm{Mg}_{3} \mathrm{Bi}_{2}$ is a superionic conductor in $\beta$ phase that Barnes et al. [11] and Howells et al. [12] had done neutron diffraction experiments on $\beta-\mathrm{Mg}_{3} \mathrm{Bi}_{2}$ and concluded that they had observed mobile $M g^{2+}$ ions with $B i^{3-}$ ions forming the rigid lattice. The electronic and structural properties of liquid $M g--B i$ alloy are investigated by Guo et al. [13]. First principles molecular dynamics simulation of liquid $\mathrm{Mg}_{3} \mathrm{Bi}_{2}$ has also been calculated by Wijs [9] and $\mathrm{HaO}$ et al. [14]. Structural and elastic properties of solid $\mathrm{Mg}_{3} \mathrm{Bi}_{2}$ is calculated by Zhou et al. [15] and Sedighi et al. [16] by first-principles calculations.

Despite these studies, there are a limited number of studies on $\mathrm{Mg}_{3} \mathrm{Bi}_{2}$ and for this reason only some parts of the physical properties exist. To our knowledge, static, dynamic, mechanical and transport properties below the

* corresponding author; e-mail: filizkayadibi@gmail.com melting point are insufficient in the literature for both with simulation and experimental study. In this study, we have performed classical molecular dynamics simulations mainly to investigate these properties such as lattice constants, radial distribution functions, mean square displacements, diffusion coefficient, heat capacity, bulk modulus, shear modulus and elastic constants. A standard molecular dynamics simulation code (MOLDY) [17] is used to calculate properties of $\mathrm{Mg}_{3} \mathrm{Bi}_{2}$ with a newly parametrized potential.

\section{Computational technique and molecular dynamics simulation}

\subsection{Computational technique}

In this study, we have developed semi-empirical potential parameters for the Born-Mayer-Huggins (BMH) form with Coulomb contribution that is given in Eq. (1):

$$
\varphi_{i j}\left(r_{i j}\right)=\frac{Z_{i} Z_{j} e^{2}}{r_{i j}}+A \exp \left(-\frac{r_{i j}}{\rho}\right)-\frac{C}{r_{i j}^{6}} .
$$

The first term is the Coulomb interaction between the effective charges $Z_{i j}$. Second term models the repulsion between the ions arising from the Pauli exclusion principle. Third term is the dipole-dipole contribution. $r_{i j}$ is the distance between ions. Potential parameters are given in Table I.

TABLE I

The values of parameters in the potential given for Eq. (1) used for the MD simulations.

\begin{tabular}{c|c|c|c|c|c}
\hline \hline \multicolumn{2}{l|}{} & $\mathrm{A}[\mathrm{eV}]$ & $\rho[\mathrm{A}]$ & $C\left[\mathrm{eV} \mathrm{A}^{6}\right]$ & $Z$ \\
\hline $\mathrm{Bi}$ & $\mathrm{Bi}$ & 0.0 & 1.0 & 0.0 & -2.04 \\
$\mathrm{Bi}$ & $\mathrm{Mg}$ & 1113.544811 & 0.38333 & 0.0 & 0.0 \\
$\mathrm{Mg}$ & $\mathrm{Mg}$ & 9547.96 & 0.21916 & 0.0 & 1.36
\end{tabular}

Parameters were adjusted to reproduce experimental data like lattice constants, bulk modulus and elastic constants where lattice constant is an experimental value $[10,18]$ and elastic properties are from firstprinciple calculations [15, 16]. Moreover, the Bredig 
phase transition of $\mathrm{Mg}_{3} \mathrm{Bi}_{2}$ has been taken into account while obtaining potential parameters. Experimental measurements show that the $\alpha \rightarrow \beta$ transition takes place at $976 \mathrm{~K} \mathrm{[12]} \mathrm{and} \mathrm{melting} \mathrm{at} 1094 \mathrm{~K}$, which is another critical point for the potential parameter development.

\section{2. $M D$ simulation}

Molecular dynamics simulation of $\mathrm{Mg}_{3} \mathrm{Bi}_{2}$, consisting of 6655 particles with $3993 \mathrm{Mg}^{2+}$ ions and 2662 $\mathrm{Bi}^{3-}$ ions, were performed at different temperatures up to $1600 \mathrm{~K}$ approximately $500 \mathrm{~K}$ above the experimental melting temperature. $\mathrm{Mg}_{3} \mathrm{Bi}_{2}$ has the hexagonal anti- $\mathrm{La}_{2} \mathrm{O}_{3}$ type structure (space group $P 3 \mathrm{~m} 1$ ) with lattice parameters of 4.674 and 7.416 , respectively [18]. $11 \times 11 \times 11$ simulation box with periodic boundary conditions is used to demonstrate the system. Simulations have been carried out by the molecular dynamics program, MOLDY [17]. We have used the Beeman algorithm, which integrates the Newton equation of motion, find positions and velocities of ions with the system time step $\Delta t=1 \mathrm{fs}$, here the total simulation time is $100 \mathrm{ps}$. When the system reached equilibrium after $30 \mathrm{ps,} \mathrm{we} \mathrm{av-}$ erage over the following $70 \mathrm{ps}$ to calculate physical properties. The Ewald sum technique is used to obtain longrange Coulomb interactions. Our MD calculations have been performed in two parts. In the first part, initial configuration of the system is the crystal structure and the calculations are carried out from $300 \mathrm{~K}$ to $1600 \mathrm{~K}$ by restarting from the previous runs with $50 \mathrm{~K}$ intervals. In the second part, the system has been started as a liquid structure at $1100 \mathrm{~K}$. Here the initial configuration is generated such that it is not periodic in three dimensions but with a regular minimum separation between center of masses. Molecules are placed at an interval that is called as skew start method. Second procedure is used in order to compare our radial distribution function with first-principle calculations where they also started their simulation with same initial configuration. All the calculations except the second procedure were performed in the constant pressure-temperature ensemble applying the Nosé-Hoover thermostat and the Parrinello and Rahman (P-R) constant stress method to control the temperature and pressure. Liquid structure simulations were performed in NVE ensemble for the density $5.467 \mathrm{~g} / \mathrm{cm}^{3}$.

\section{Results and discussion}

\subsection{Radial distribution function}

The radial distribution function, RDF, also called pair distribution function is one of the most important tools defining the structure of a system. The radial distribution function describes how the density changes as a function of distance from a reference particle, which is defined as following in Eq. (2):

$$
g(r)=\frac{\Delta n(r)}{\rho 4 \pi r^{2} \Delta r}
$$

$\Delta n(r)$ is the mean number of atoms at a distance between $r$ and $r+\Delta r$ from a given atom. $\rho$ is the mean atom density and $\Delta r$ is the width of the shell.
The radial distribution functions of $\mathrm{Mg}_{3} \mathrm{Bi}_{2}$ at $300 \mathrm{~K}$ and $1000 \mathrm{~K}$ are calculated. At $300 \mathrm{~K}$, initial configuration is a crystal and for every $50 \mathrm{~K}$ interval restarted from the previous run up to $1000 \mathrm{~K}$. For $1100 \mathrm{~K}$, simulation is initiated from liquid configuration.

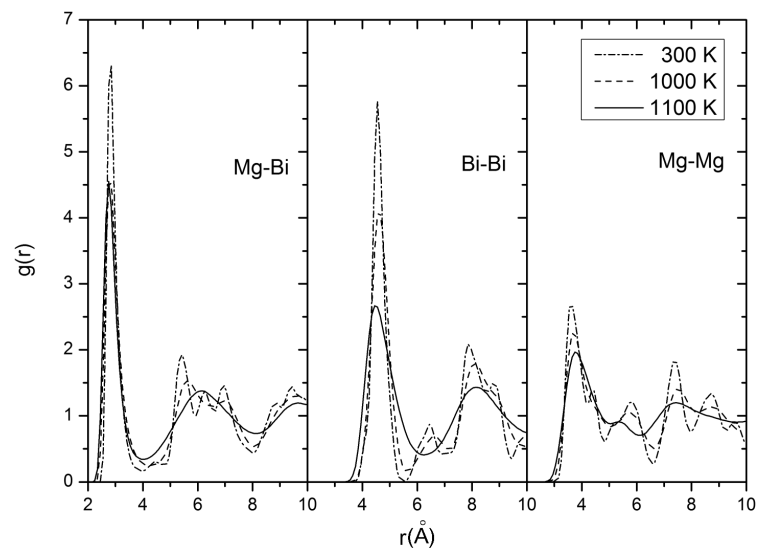

Fig. 1. The radial distribution functions of $\mathrm{Mg}_{3} \mathrm{Bi}_{2}$ at 300,1000 and $1100 \mathrm{~K}$.

In Fig. 1, the radial distribution functions of $\alpha-\mathrm{Mg}_{3} \mathrm{Bi}_{2}$, $\beta-\mathrm{Mg}_{3} \mathrm{Bi}_{2}$ and liquid $\mathrm{Mg}_{3} \mathrm{Bi}_{2}$ are given from our MD calculations at 300, 1000, and $1100 \mathrm{~K}$, respectively. As shown in the graph, all the peaks are narrow and sharp in the solid phase and as temperature increases to the liquid phase they gradually become shorter and wider because of the larger thermal oscillations of particles from their lattice points. At $1000 \mathrm{~K}$, the $g_{\mathrm{MgMg}}(r)$ is rather broad and lower with less pronounced oscillations at larger $r$ as compared to $g_{\mathrm{BiBi}}(r)$ which indicates that the cations are less structured than the anions with a higher $\mathrm{Mg}$ ion mobility like in the liquid phase so the $g_{\mathrm{BiBi}}(r)$ has a high degree of correlation as expected. This is a characteristic feature of a superionic (fast-ion) conductor [19] in which magnesium ions are the mobile ions with the bismuth ions forming the rigid lattice. Moreover we can also observe the first nearest neighbor separations between $\mathrm{Mg}-\mathrm{Mg}, \mathrm{Bi}-\mathrm{Bi}$, and $\mathrm{Mg}-$ $\mathrm{Bi}$ at $300 \mathrm{~K}$ and $1000 \mathrm{~K}$ from Fig. 1. First coordination peaks indicate the first nearest neighbor separations at $3.62,4.54$, and $2.84 \AA$ at $300 \mathrm{~K}$ for $\mathrm{Mg}-\mathrm{Mg}, \mathrm{Bi}-\mathrm{Bi}$, and $\mathrm{Mg}-\mathrm{Bi}$, respectively, and it is also obtained from the same figure that values are $3.73,4.59$, and $2.80 \AA$ at $1000 \mathrm{~K}$, which agrees with the experimental values $3.75,4.58$, and $2.80 \AA$ [11]. Additionally coordination number of $\mathrm{Mg}-\mathrm{Bi}$ is calculated from Fig. 1 at $300 \mathrm{~K}$ with Eq. (3):

$$
\left\langle n_{i j}(r) \Delta(r)=4 \pi r^{2} \Delta r \rho_{j} g_{i j}(r)\right\rangle \text {. }
$$

Number of $j$ particles around $i$ particles in a shell of $\Delta r$ is given by $n_{i j}(r) \Delta r . \rho_{j}$ is the number density of $j$ particles in MD cell. We have calculated $\mathrm{Bi}-\mathrm{Mg}$ coordination number where $\mathrm{Bi}$ atoms are surrounded by $7 \mathrm{Mg}$ atoms and this result is in agreement with experimental data [20].

In Fig. 2, the radial distribution functions of liquid $\mathrm{Mg}_{3} \mathrm{Bi}_{2}$ at $1100 \mathrm{~K}$ are compared with the first principle 


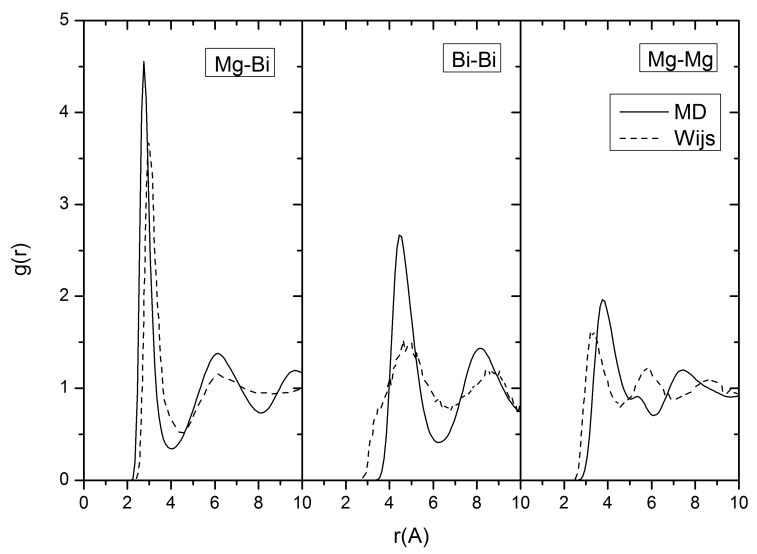

Fig. 2. The radial distribution functions of liquid $\mathrm{Mg}_{3} \mathrm{Bi}_{2}$ at $1100 \mathrm{~K}$. Solid line represents our MD calculations and dashed line is the first principles calculations [9].

calculations [9] and these calculations are in good agreement with the Weber experimental data [21]. Both of the simulation results have the initial configuration of liquid structure where density is $\mathrm{Mg}_{3} \mathrm{Bi}_{2}$.

\subsection{Lattice parameters, density and thermal expansion}

We have obtained the lattice parameters of $\mathrm{Mg}_{3} \mathrm{Bi}_{2}$ with a constant pressure calculation, which is allowing the atoms in the unit cell relax to zero strain where it minimizes the total energy of the system. BFGS energy minimization strategy is used to search stationary point that is implanted in GULP program. $T=0 \mathrm{~K}$ lattice parameters are $a_{0}=4.61, c_{0}=7.2389$ and unit cell angles are $\theta_{1}=89.99, \theta_{2}=90.00$ and $\gamma=119.99$. Cohesive energy per molecule is $4862.499 \mathrm{~kJ} / \mathrm{mol}$. Zhou et al. [15] have found the cohesive energy $2575.36 \mathrm{~kJ} / \mathrm{mol}$ from first principle calculations. The calculated lattice parameters at room temperature are in fairly good agreement with available theoretical and experimental data $[10,15$, $16,18,22-24]$. All values of lattice parameters are given in Table II. We have also calculated the lattice parameters as a function of temperature. It has already been known that $\alpha-\mathrm{Mg}_{3} \mathrm{Bi}_{2}$ does not melt directly but undergoes a transition to $\beta$ phase at $976 \mathrm{~K}$ and $\beta$-phase melts at $1094 \mathrm{~K}$ [11]. The constant stress method of Parrinello and Rahman dynamics is used in the MD simulation. With this method, the system's shape can change and therefore both pressure and stress can be controlled. Eventually, lattice parameters in each dimension and each unit cell angles may change.

The variations of lattice constants $a_{1}, a_{2}, c$ and the angles $\theta_{1}, \theta_{2}, \gamma$ with temperature are shown in Figs. $3-$ 5 . The variation of density with temperature is given in Fig. 6. It is a well-known fact that MD overestimates the melting point of one phase crystal system. For this reason we have estimated the melting point about $1250-1300 \mathrm{~K}$. This melting point will also be observed in other temperature dependent physical parameters through this article. Here in Figs. 3-5, structural

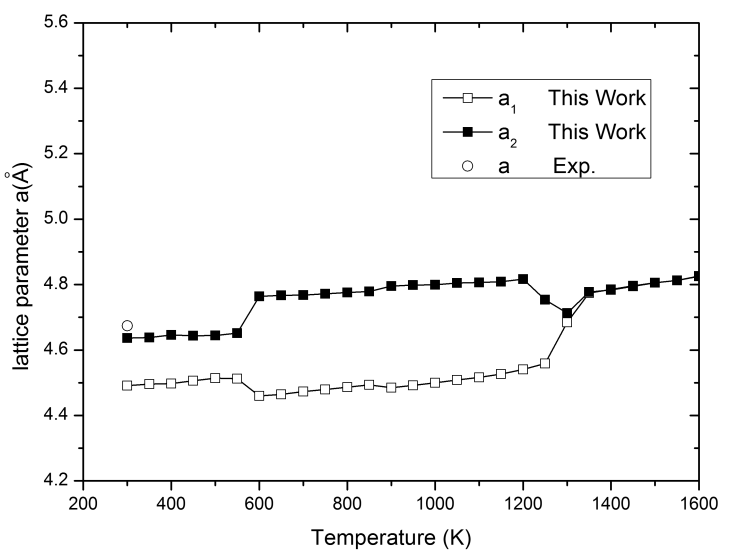

Fig. 3. Variation of lattice constant $a$ with temperature.

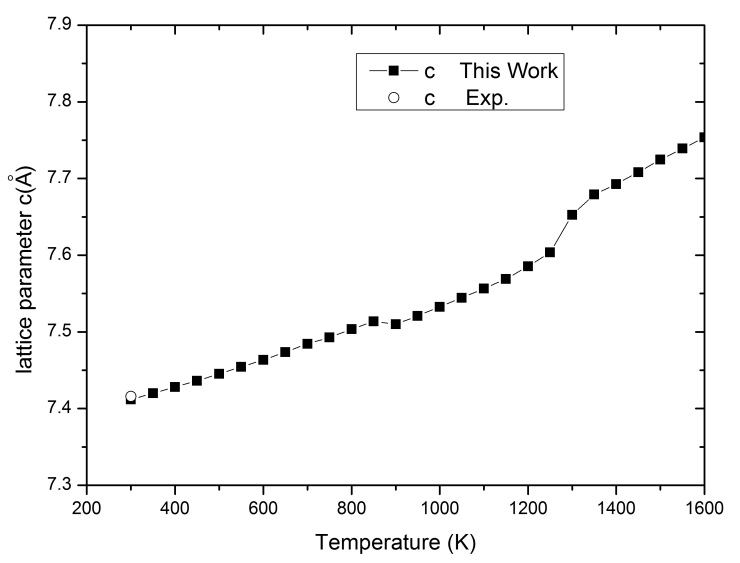

Fig. 4. Variation of lattice parameter $c$ with temperature.

changes are monitored up to melting point. At around 500-600 K, lattice parameters $a_{1}, a_{2}$ for both directions change about $2 \%$. At $850-900 \mathrm{~K}$, lattice parameter $a_{1}, a_{2}, c$ changes about $0.5 \%$. Finally all three parameters change at MD melting point $1250 \mathrm{~K}-1300 \mathrm{~K}$. These changes are also observed for angles $\theta_{1}, \theta_{2}, \gamma$ in Fig. 5. These variations below the melting point may

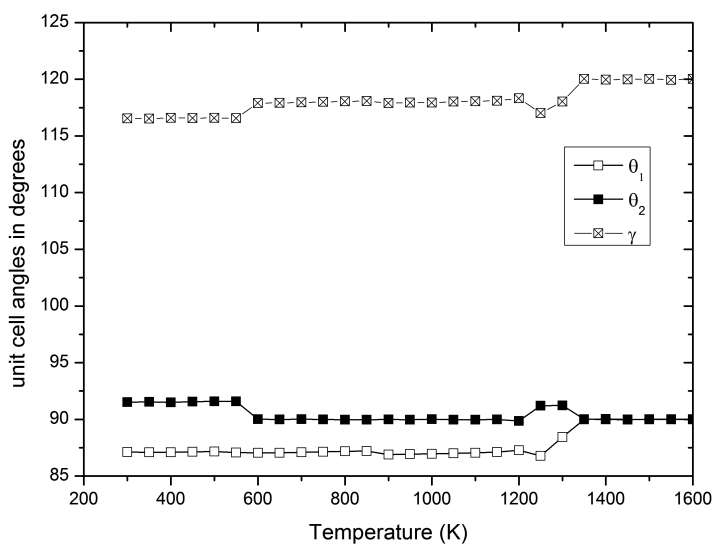

Fig. 5. Variation of unit cell angles with temperature. 
TABLE II

Lattice constants (in $\AA$ ) of $\alpha-\mathrm{Mg}_{3} \mathrm{Bi}_{2}$.

\begin{tabular}{c|c|c|c}
\hline \hline \multicolumn{4}{c}{ Lattice constants } \\
\hline & $a$ & $c$ & $c / a$ \\
\hline This work & 4.61 & 7.24 & 1.571 \\
{$[10]$} & 4.666 & 7.401 & 1.586 \\
{$[15]$} & 4.67 & 7.41 & 1.586 \\
{$[16]$} & 4.66 & 7.333 & 1.573 \\
{$[18]$} & 4.674 & 7.416 & 1.586 \\
{$[22]$} & 4.671 & 7.403 & 1.584 \\
{$[23]$} & 4.67 & 7.40 & 1.584 \\
{$[24]$} & 4.65 & 7.38 & 1.587
\end{tabular}

be attributed to the $\alpha \rightarrow \beta$ phase transition. In Fig. 6 , calculated density up to $1600 \mathrm{~K}$ compared with experimental data. At $300 \mathrm{~K}$, density of $\mathrm{Mg}_{3} \mathrm{Bi}_{2}$ is in good agreement with experimental data. Again, it could also be observed that experimental melting point density of $\mathrm{Mg}_{3} \mathrm{Bi}_{2}(1100 \mathrm{~K})$ and MD simulation melting point density of $\mathrm{Mg}_{3} \mathrm{Bi}_{2}(1250-1300 \mathrm{~K})$ are also in good agreement. We can conclude that our MD simulation calculations with BMH potential display the structural distortions in phase transitions at correct temperatures.

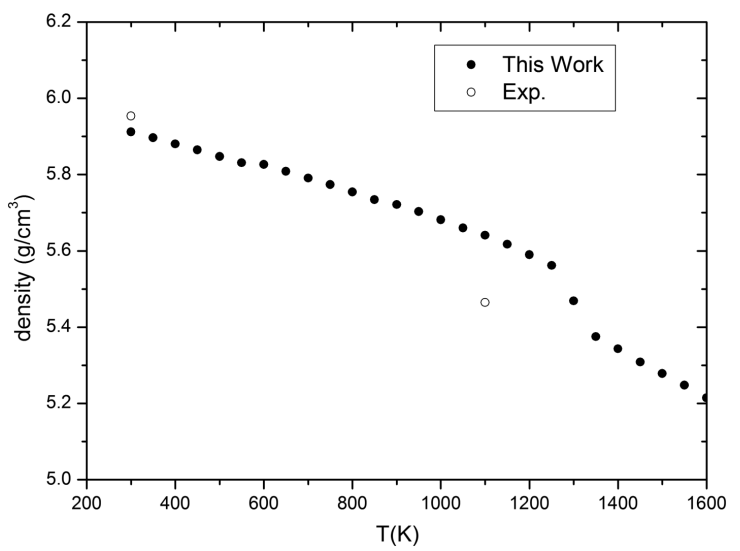

Fig. 6. Variation of density with temperature.

\subsection{Diffusion of $\mathrm{Mg}^{2+}$ and $\mathrm{Bi}^{3-}$ sublattice}

The mean square displacement (MSD) data of atoms in a simulation contains information on the atomic diffusivity. If the system is solid, the kinetic energy is not sufficient enough for atoms to reach a diffusive behavior, in such a case the slope of MSD is zero. Nevertheless if the system is not solid (e.g. liquid) then the MSD will change in time and the slope is different than zero where ions diffuse through the lattice. Mean square displacement of ionic species is a way to calculate the diffusion as follows:

$$
\left\langle r_{\alpha}^{2}(t)\right\rangle=\frac{1}{3 N_{\alpha}} \sum_{i \alpha}^{N \alpha}\left\langle\left|r_{i \alpha}(t)-r_{i \alpha}(0)\right|^{2}\right\rangle,
$$

where $r_{i \alpha}(t)$ is the position of the $i$-th ion at time $t$. The mean square displacements of $M g^{2+}$ and $B i^{3-}$ ions from the room temperature up to $1300 \mathrm{~K}$ for $11 \times 11 \times 11$ supercell are presented in Fig. 7 as a function of time. It has been reported that $\alpha-\mathrm{Mg}_{3} \mathrm{Bi}_{2}$ in solid state has a second order structural transition to the superionic phase at nearly $976 \mathrm{~K}$ [11]. Superionic phase transition can be explained as: an order-disorder transition starts by the collapse of $M g^{2+}$ sub-lattice and $M g^{2+}$ ions behave like a liquid while $B i^{3-}$ ions are still solid. In such a case the behaviors of ions correspond to the superionic phase transition. Diffusion for $M g^{2+}$ ions are almost zero up to $1000 \mathrm{~K}$. Above this point $\mathrm{Mg}$ diffusion starts and $\mathrm{Bi}$ also shows signs of mobility that we do not expect at this temperature. This could be commented that melting and superionic phase transition temperatures are relatively too close to each other about $100 \mathrm{~K}$ for a MD simulation. Otherwise, if there would be a larger gap between transition and melting point temperature, we should observe $\mathrm{Bi}$ ions totally immobile, preserving its rigid sublattice at the superionic transition temperature. It is a generally known issue that MD simulations overestimate the melting temperature [25]. Even there exist no general rules that we would deduce from the previous studies that melting temperature of solid MD box is about $20 \%-30 \%$ above the experimental data [26]. In this study, melting point of the $\mathrm{Mg}_{3} \mathrm{Bi}_{2}$ molecule is also overestimated. At $1300 \mathrm{~K}$, in Fig. 7, we could clearly observe both sublattices totally collapsed. From this we could deduce that melting temperature is about $1250-1300 \mathrm{~K}$ where the experimental value is $1094 \mathrm{~K}$.

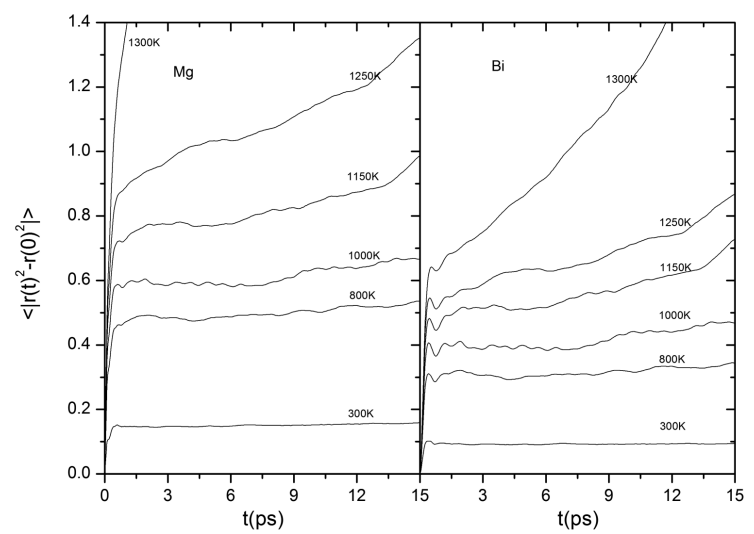

Fig. 7. Mean square displacements of $\mathrm{Mg}^{+2}$ and $B i^{-3}$ ions.

The diffusion constant of ions using the Einstein relation can be calculated from the gradient of the MSD graph

$$
\left\langle\left|r_{i \alpha}(t)-r_{i \alpha}(0)\right|^{2}\right\rangle=6 D t
$$

or diffusion coefficient may also be calculated from the velocity auto-correlation function (VACF). Following relation shows VACF function

$$
\begin{aligned}
& C(t)=\left\langle\boldsymbol{V}_{i}\left(t_{0}\right) \cdot \boldsymbol{V}_{i}\left(t_{0}+\tau\right)\right\rangle= \\
& \frac{1}{N} \sum_{i=1}^{N} \frac{1}{t_{\max }} \sum_{t_{0}=1}^{t_{\max }} \boldsymbol{V}_{i}\left(t_{0}\right) \cdot \boldsymbol{V}_{i}\left(t_{0}+\tau\right),
\end{aligned}
$$


where $N$ is the particle number. Diffusion coefficient $(D)$ is

$$
D=\frac{1}{3} \int_{0}^{\infty} C(t) \mathrm{d} t .
$$

Diffusion coefficients of $\mathrm{Mg}_{3} \mathrm{Bi}_{2}$ molecules are shown in Fig. 8, which are calculated from VACF and MSD data, and display slightly different behavior up to $650 \mathrm{~K}$. In spite of discrepancies at low temperatures, it should be noted that this is a logarithmic plot and the values are too small when it is compared with high temperatures. Both of the calculations of diffusion coefficient increase with temperature and at about $1050 \mathrm{~K} \mathrm{Mg}$ diffusion coefficient changes anomalously when compared to $\mathrm{Bi}$. Diffusion coefficient of $\mathrm{Mg}$ ion is higher than $\mathrm{Bi}$ ion above the $\alpha \rightarrow$ $\beta$ phase transition temperature.

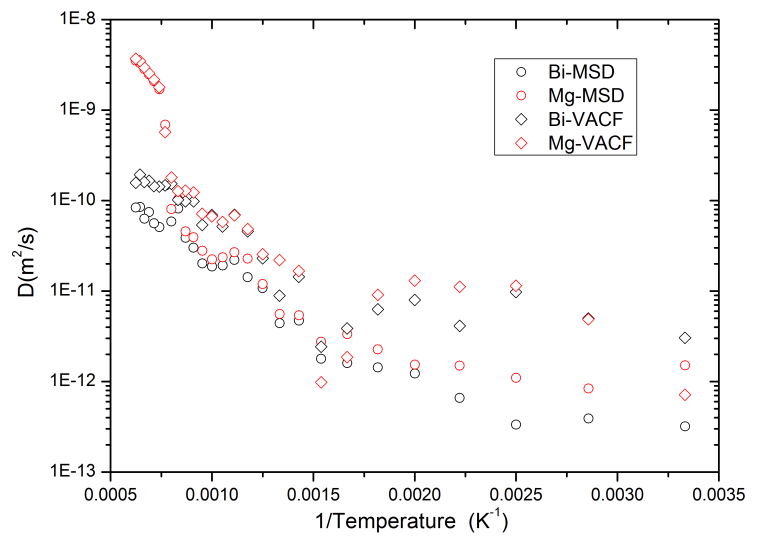

Fig. 8. An Arrhenius plot of $\mathrm{Mg}_{3} \mathrm{Bi}_{2}$ from $300 \mathrm{~K}$ up to $1600 \mathrm{~K}$

In literature there is a limited number of experimental data on diffusion of $\mathrm{Mg}_{3} \mathrm{Bi}_{2}$ as far as we know. Howells [12] had measured the diffusion coefficient of $\mathrm{Mg}$ ions by neutron scattering experiment. He concluded that the diffusion coefficient is $2.7 \pm 0.3 \times 10^{-9} \mathrm{~m}^{2} / \mathrm{s}$ for both $\beta$ and liquid phases. From Fig. 8, our estimated value of $\mathrm{Mg}$ ion diffusion coefficient for the liquid phase $\left(2.18 \times 10^{-9} \mathrm{~m}^{2} / \mathrm{s}\right.$ is in agreement with experimental data.

\subsection{Enthalpy and heat capacity}

$\alpha-\mathrm{Mg}_{3} \mathrm{Bi}_{2}$ molecule has a phase transitions from $\alpha \rightarrow \beta$ and $\beta \rightarrow$ melting point. For these reason it is a good idea to investigate energy and related properties. Enthalpy curve is a useful tool to observe phase transitions. The enthalpy change $\Delta H=H_{T}-H_{300}$ is presented in Fig. 9. A discontinuity of data indicates some abnormal phenomena are taking place like the phase transition. In Fig. 10, the specific heat at constant pressure can be determined from the variation of the internal energy of the system with temperature as

$$
C_{P}(T)=\left(\frac{\partial E}{\partial T}\right)_{P} .
$$

In Fig. 10, estimated value of heat capacity is equal to classical value of the Dulong-Petit law. As the temperature increases, the slope of heat capacity changes gently by the creation of magnesium Frenkel pairs. When we compare $\mathrm{Mg}$ and $\mathrm{Bi}$ ions from the diffusion data, $\mathrm{Mg}$ ions are inherently more mobile. As a result of these, it can be stated that much of the Frenkel pairs are Mg. Between 800-1100 K, small deviations from the classical heat capacity value can be attributed to the change of lattice parameters $a$ and $c$. From $1100 \mathrm{~K}$ to $1400 \mathrm{~K}$ there exists a rapid increase in the heat capacity data. Here, both superionic transition and melting point appear in this temperature range. One can deduce this from MSD in Fig. 7 and in general every phase change indicates itself in energy data. Here the phase transitions, $\alpha \rightarrow \beta$ $(976 \mathrm{~K})$ and $\beta \rightarrow$ melting point $(1094 \mathrm{~K})$, are so close that it can be concluded that these peaks merged into one peak.

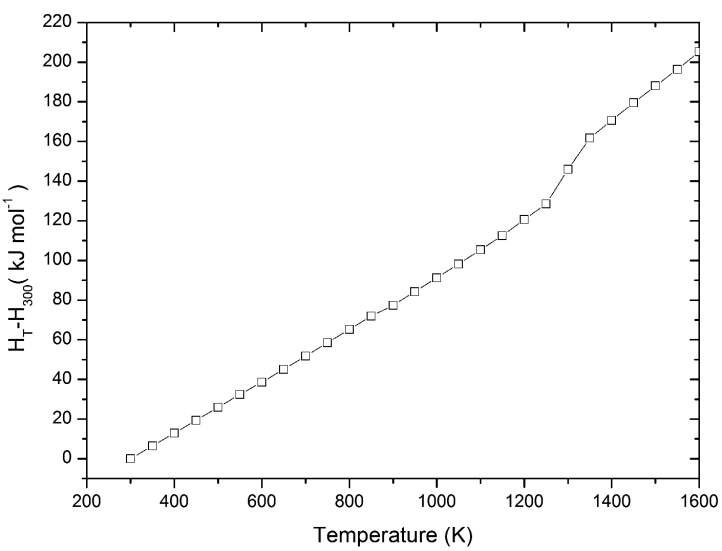

Fig. 9. Enthalpy change of $\mathrm{Mg}_{3} \mathrm{Bi}_{2}$ with temperature.

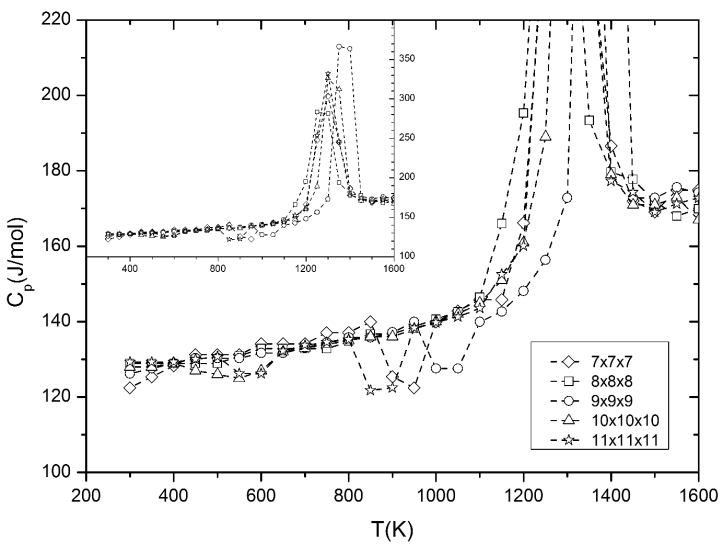

Fig. 10. Heat capacity of $\mathrm{Mg}_{3} \mathrm{Bi}_{2}$ for different supercell sizes change with temperature.

\subsection{Elastic constants, bulk modulus and shear modulus}

Hardness of a material is determined with the elastic constants, bulk and shear modules. These constants are calculated by different types of deformations of the crystal. Bulk modulus can be measured by the BirchMurnaghan equation of state. When isotropic pressure 
is applied to a material, this equation is fitted to the variation of energy or variation of pressure with the volume

$$
B=V\left(\frac{\partial^{2} U}{\partial V^{2}}\right)=-V\left(\frac{\partial P}{\partial V}\right) .
$$

$U$ is the energy, $V$ is the volume and $P$ is the pressure. Equation of state data can be easily obtained from the MD simulation by changing the volume around the minimum energy point. Bulk and shear modules can also be calculated by the combination of elastic constants

$$
\begin{aligned}
B & =\frac{1}{9}\left(C_{11}+C_{22}+C_{33}+2\left(C_{12}+C_{13}+C_{23}\right)\right), \\
G & =\frac{1}{15}\left(C_{11}+C_{22}+C_{33}+3\left(C_{44}+C_{55}+C_{66}\right)\right. \\
& \left.-C_{12}-C_{13}-C_{23}\right) .
\end{aligned}
$$

These equations are Voigt definition of bulk and shear modulus. Shear modulus describes the crystal's response to shear stress. Elastic properties are estimated from the second-order derivatives of the potential with respect to strain

$$
C_{i j}=\frac{1}{V}\left(\frac{\partial^{2} U}{\partial \varepsilon_{i} \partial \varepsilon_{j}}\right) .
$$

Here $\varepsilon$ is the deformation. Since $\alpha-\mathrm{Mg}_{3} \mathrm{Bi}_{2}$ is a noncubic material, $C_{13}, C_{33}$ should also be investigated in addition to $C_{11}, C_{12}$ and $C_{44}$. All the elastic properties are investigated by GULP program [27]. To the best of our knowledge there are only two measured values of $\alpha-\mathrm{Mg}_{3} \mathrm{Bi}_{2}$ bulk modulus $[15,16]$. Both of them are firstprinciple calculations and different from each other by about $15 \%$. Shear and elastic modulus data are only compared with one calculated data [15]. Most of our results, except $C_{3} 3$ and $C_{4} 4$, are in good agreement with quantum chemical calculation that is given in Table III.

\section{TABLE III}

Elastic properties of $\mathrm{Mg}_{3} \mathrm{Bi}_{2}$.

\begin{tabular}{c|c|c}
\hline \hline & Ab initio & Present work \\
\hline bulk modulus [GPa] & $45.81[15] /$ & 39.03 \\
shear modulus [GPa] & $79.8[16]$ & \\
$C_{11}$ [GPa] & $64.26[15]$ & 7.92 \\
$C_{33}[\mathrm{GPa}]$ & $103.64[15]$ & 69.77 \\
$C_{12}[\mathrm{GPa}]$ & $36.59[15]$ & 41.55 \\
$C_{13}[\mathrm{GPa}]$ & $39.53[15]$ & 25.67 \\
$C_{44}[\mathrm{GPa}]$ & $4.04[15]$ & 22.59
\end{tabular}

\section{Conclusions}

In this study, we have investigated $\mathrm{Mg}_{3} \mathrm{Bi}_{2}$ from room temperature to $1600 \mathrm{~K}$ by molecular dynamics simulation with newly developed potential parameters of BMH type potential. Lattice parameters, elastic constants, bulk modulus and shear modulus are the main observables and reference data while we are developing the potential parameters. As we increase the temperature, phase transitions, $\alpha \rightarrow \beta$ and $\beta \rightarrow$ melting point, are also taken into account.
Lattice parameters are calculated as $a=4.61 \AA$ and $c=7.24 \AA$ and compared with a considerable amount of experimental and first principle data $[10,15,16,18$, 22-24] which are in agreement with these results (Table II). As the temperature increases from room temperature to $1600 \mathrm{~K}$, constant stress method of Parrinello and Rahman dynamics is used where lattice parameters change in each dimension so that lattice constant $a_{1}$ and $a_{2}$ diverge from each other and angles do not maintain their $\alpha=90^{\circ}, \beta=90^{\circ}, \gamma=120^{\circ}$ values. Among the NPT ensembles constant stress method of $\mathrm{P}-\mathrm{R}$ is chosen in order to avoid uniform dilation or contraction of $\mathrm{Mg}_{3} \mathrm{Bi}_{2}$ crystal structure where it is experimentally known that as the temperature increases hexagonal $\mathrm{Mg}_{3} \mathrm{Bi}_{2}$ structure changes into $\beta-\mathrm{Mg}_{3} \mathrm{Bi}_{2}$ structure by the non-uniform change of lattice parameters and angles. Experimental density at room temperature is $5.95 \mathrm{~g} / \mathrm{cm}^{3}$ and $5.465 \mathrm{~g} / \mathrm{cm}^{3}$ at melting point. Our results are $5.91 \mathrm{~g} / \mathrm{cm}^{3}$ and $5.468 \mathrm{~g} / \mathrm{cm}^{3}$, respectively. Room temperature and liquid phase densities are in agreement with experimental data but it should be noted that MD melting point temperature is overestimated about $200 \mathrm{~K}$ which is normal for one phase supercell simulation box system. Radial distribution functions of liquid $\mathrm{Mg}_{3} \mathrm{Bi}_{2}$ are also compared with first-principle calculations. Except Bi-Bi first peak, they roughly have the same behavior.

RDF peak positions are in good agreement with experimental values for both room temperature and melting point data. Howells commented on his empirical diffusion coefficient value of $\mathrm{Mg}$ ion which it is the same both for the superionic phase and liquid phase whereas we think this should be reconsidered again because temperature difference between these two points are $100 \mathrm{~K}$ and diffusion constant could not be the same. Nevertheless, liquid diffusion coefficient derived both from mean square displacement and velocity auto-correlation functions for liquid phase confirm that our results are compatible with the experimental value. Sedighi [16] and Zhou [15] calculated the bulk modulus from first principle with a certain amount of value (15\%) different from each other, which makes these values somewhat unreliable and these data needs to be verified by experimental methods. Our data significantly resembles the latest first principle calculation by Sedighi [16]. Zhou et al. also obtain values of elastic properties, like elastic constants, shear modulus. Our calculated values are in agreement with shear modulus, $C_{11}, C_{12}$ and $C_{13}$. All these results display the features of promising classical interaction potential parameters for $\mathrm{MD}$ simulation of $\mathrm{Mg}_{3} \mathrm{Bi}_{2}$ which is valid for a broad temperature range.

\section{References}

[1] B.L. Mordike, T. Ebert, Mater. Sci. Eng. A 302, 37 (2001).

[2] A. Luo, M.O. Pekgüleryüz, J. Mater. Sci. 29, 5259 (1994). 
[3] H. Honda, H. Sakaguchi, I. Tanaka, T. Esaka, J. Power Sources 123, 216 (2003).

[4] T. Kajikawa, N. Kimura, T. Yokoyama, Thermoelectrics ICT 2003, 305 (2003).

[5] P. Singh, K.K. Sarkar, Solid State Commun. 55, 439 (1985).

[6] L.W. Watson, C.A.W. Marshall, C.P. Cardoso, J. Phys. F Met. Phys. 14, 113 (1984).

[7] G. Yuan, Y. Sun, W. Ding, Mater. Sci. Eng. A 308, 38 (2001).

[8] G. Yuan, M. Liu, W. Ding, I. Akihisa, Mater. Sci. Eng. A 357, 314 (2003).

[9] G.A. de Wijs, G. Pastore, A. Selloni, W. van der Lugt, J. Phys. Condens. Matter 8, 1879 (1996).

[10] Crystal Structures, Ed. R.W.G. Wyckoff, 2nd ed., Vol. 1, Wiley, New York 1963.

[11] A.C. Barnes, C. Guo, W.S. Howells, J. Phys. Condens. Matter 6, L467 (1994)

[12] W.S. Howells, A.C. Barnes, M. Hamilton, Physica B 266, 97 (1999).

[13] C. Guo, A.C. Barnes, W.S. Howells, J. Phys. Condens. Matter 8, 10823 (1996).

[14] Q.H. Hao, Y.W. You, X.S. Kong, C.S. Liu, Int. J. Mod. Phys. B 27, 1350011 (2013).

[15] D.W. Zhou, J.S. Liu, S.H. Xu, P. Peng, Physica B 405, 2863 (2010).
[16] M. Sedighi, B.A. Nia, H. Zarringhalam, R. Moradian, Eur. Phys. J. Appl. Phys. 61, 10103 (2013).

[17] K. Refson, Comput. Phys. Commun. 126, 310 (2000).

[18] Structure and Properties of Inorganic Solids, Ed. F.S. Galasso, Pergamon Press, Oxford 1970 p. 157.

[19] Physics of Superionic Conductors, Ed. M.B. Salamon, Springer, Berlin 1979.

[20] Structural Inorganic Chemistry, Ed. A.F. Wells, 4th ed., Clarendon Press, Oxford 1975.

[21] M. Weber, S. Steeb, P. Lamparter, Z. Nat.forsch. A 34, 1398 (1979).

[22] E. Zintl, E. Husemann, Z. Phys. Chem. B 21, 138 (1933).

[23] Y. Imai, A. Watanabe, J. Mater. Sci. 41, 2435 (2006).

[24] L.G. Sevast'yanova, O.V. Kravchenko, O.K. Gulish, V.A. Stupnikov, M.E. Leonova, M.G. Zhizhin, Inorg. Mater. 42, 863 (2006).

[25] F. Ercolessi, S. Iarlori, O. Tomagnini, E. Tosatti, X.J. Chen, Surf. Sci. 251/252, 645 (1991).

[26] J.R. Morris, C.Z. Wang, K.M. Ho, C.T. Chan, Phys. Rev. B 49, 3109 (1994).

[27] J.D. Gale, A.L. Rohl, Mol. Simul. 29, 291 (2003). 